\title{
Optimal Placement of Relay Infrastructure in Heterogeneous Wireless Mesh Networks by Bender's Decomposition
}

\author{
Aaron So \\ Department of Electrical and Computer \\ Engineering \\ University of Toronto, Toronto, Ontario, Canada \\ aaronso@comm.utoronto.ca
}

\begin{abstract}
Fixed Broadband Wireless Access (FBWA) technology is designed to serve as a wireless DSL replacement technology to provide broadband access in underserved areas where no other access technology exists. Due to the enormousness of the target service area, relay equipment play an important role in such networks, and the installation and maintenance cost of the network is directly proportional to the cost of the relay equipment. To minimize the network operational cost, we develop an optimization framework which computes the minimum number of relay stations and their placement in the network such that the demands from the end users are met.
\end{abstract}

\section{INTRODUCTION}

Broadband wireless has long held the promise of delivering a wide range of cost-effective data services. With the publication of a comprehensive industry standard, namely IEEE 802.16, and the introduction of microchips based on that standard by leading semiconductor companies, broadband wireless has gained the maturity and is ready to unleash its full potential. However, at this point, broadband wireless is still a divergent, even disruptive, technology, and wireline solutions such as DSL constitute the mainstream. For this reason, innovative yet cost effective strategies have to be invented to make broadband wireless more attractive to broadband Internet service providers.

Due to the different propagation characteristics of radio waves in the lower- and upper-microwave regions, IEEE 802.16 standard requires two separate physical layer specifications. Lower frequency signals can penetrate walls and deflect from obstacles, while higher frequency transmissions must meet strict line-of-sight requirements. However, the advantage of using high frequency bands is an abundance of bandwidth. Moreover, communication in such a high fre-

Permission to make digital or hard copies of all or part of this work for personal or classroom use is granted without fee provided that copies are not made or distributed for profit or commercial advantage and that copies bear this notice and the full citation on the first page. To copy otherwise, to republish, to post on servers or to redistribute to lists, requires prior specific permission and/or a fee.

QShine '06, August 7-9, 2006 Waterloo, Ontario, Canada.

Copyright 2006 ACM X-XXXXX-XX-X/XX/XX ...\$5.00.

\author{
Ben Liang \\ Department of Electrical and Computer \\ Engineering \\ University of Toronto, Toronto, Ontario, Canada \\ liang@comm.utoronto.ca
}

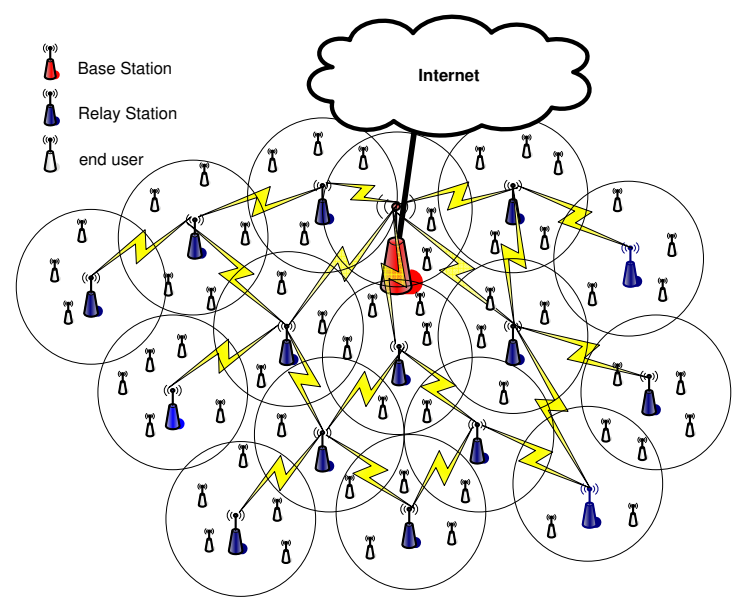

Figure 1: Heterogeneous Wireless Mesh Network.

quency spectrum facilitates the efficient use of directional antennae, which almost eliminates the effect of interference. This intrinsic property of IEEE 802.16 technology makes it ideal for a heterogeneous architecture.

An example of such architecture is illustrated in Fig. 1. In this networks, there is a base station wired to the ISP network, and this base station is assigned to serve users in a particular geographic area. Because of the size of the coverage area, several relay stations (RSs) are installed, for example, on the rooftops of end users, in the network to relay traffic from and to the base station. By using directional antennae, the bandwidth abundant high frequency spectrum is used to form a backbone network. The lower spectrum, on the other hand, is used by the base station and RSs to communicate with the end users and form the corresponding local network. The focus of this work is to minimize the number of RSs used in the mesh network while maintaining the pre-specified uplink and downlink demands of the end users.

The rest of this paper is organized as follows. In Section 2 , we review the related works on equipment placement in wireless networks. In Section 3, we define our relay station placement problem mathematically, and describe an optimization solution. In Section 4, we show the effects of the number of local channels on the optimal number and place- 
ment of RSs. Finally, concluding remarks are given in Section 5 .

\section{RELATED WORKS}

The problem of wireless network equipment placement has been addressed in several works. In the context of sensor networks, the optimal number and placement of sensors which maximize the network lifetime are discussed in [3]. In [5], we proposed an Lagrangian approach to compute the optimal placement of a fixed number of relay nodes, which relay traffic in a two-hop fashion, to improve throughput in a wireless local area network. In the context of community mesh networks, integration techniques were developed in [1] to minimize the number of access points in a mesh network to reduce wiring cost, while maintaining QoS constraints from all nodes. A similar problem was discussed in [2] under different link and network models. From the results of these works, we learn that the placement of wireless network equipments can have significant impact on network performance. To the best of our knowledge, there is no existing work address the problem of relay equipment placement in community wireless mesh network, which is the question we investigate in this work.

\section{SYSTEM MODEL AND OPTIMIZATION}

Suppose there are $N$ users and one base station in the system, and they are represented by the set $V=\{0,1, \ldots, N\}$, where the base station is represented by the index 0 . We can use the set $V$ to form two directed-complete graphs: Backbone and Local. For each complete graph, the link weight from node $i$ to node $j$ represents the capacity in terms of bit per second from node $i$ to node $j$ using a particular technology. That is, $B C_{i j}$ and $L C_{i j}$ represent the capacity from node $i$ to node $j$ by using the backbone and local technologies respectively. Furthermore, for each user $i$, there is a pre-specified uplink demand, $u_{i}$, and downlink demand, $d_{i}$.

Given the above as the input to our problem, we define the following decision variables.

$X_{i}=\left\{\begin{array}{lll}1 & \text { if an RS is installed at node } i & 0<i \leq N \\ 0 & \text { otherwise }\end{array}\right.$

$$
\begin{aligned}
f_{i j}^{d}= & \text { downlink flow from node } i \text { to node } j \text { by using } \\
& \text { backbone technology (bps) } i, j \in[0, N], i \neq j \\
f_{i j}^{u}= & \text { uplink flow from node } i \text { to node } j \text { by using } \\
& \text { backbone technology (bps) } i, j \in[0, N], i \neq j \\
h_{i j}^{d}= & \text { downlink flow from node } i \text { to node } j \text { by using } \\
& \text { local technology (bps) } i, j \in[0, N] \\
h_{i j}^{u}= & \text { uplink flow from node } i \text { to node } j \text { by using } \\
& \text { local technology (bps) } i, j \in[0, N] \\
v_{i}^{d}= & \text { amount of downlink demand deficit at node } i \\
v_{i}^{u}= & \text { amount of uplink demand deficit at node } i
\end{aligned}
$$

Note that all input and decision variables are non-negative. Moreover, we define $X_{0}=1$ since the base station is always present. Next, we formulate our problem as a mixed integer program.

\subsection{Optimization Formulation}

Our goal is to minimize the number of RSs in the system and the penalty imposed by the demand violations. The optimization formulation is as follows:

$$
\begin{array}{ll}
\min _{\mathbf{X}, \mathbf{v}}: \quad & C \sum_{i=1}^{N} X_{i}+P \sum_{i=1}^{N} v_{i}^{u}+v_{i}^{d} \\
\text { s.t. } \quad & \sum_{i=1}^{N} f_{i 0}^{u}+\sum_{i=1}^{N} h_{i 0}^{u}=\sum_{i=1}^{N} \sum_{j=0}^{N} h_{i j}^{u} \\
& \sum_{i=1}^{N} f_{0 i}^{d}+\sum_{i=1}^{N} h_{0 i}^{d}=\sum_{i=0}^{N} \sum_{j=1}^{N} h_{i j}^{d} \\
& \sum_{j=0, i \neq j}^{N} f_{j i}^{u}+\sum_{j=1}^{N} h_{j i}^{u}=\sum_{j=0, i \neq j}^{N} f_{i j}^{u} \\
& i=1,2, \ldots, N \\
& \sum_{j=0, i \neq j}^{N} f_{j i}^{d}-\sum_{j=1}^{N} h_{i j}^{d}=\sum_{j=1, i \neq j}^{N} f_{i j}^{d} \\
& i=1,2, \ldots, N \\
& \sum_{j=0}^{N} h_{i j}^{u}+v_{i}^{u} \geq u_{i} \quad i=1,2, \ldots, N \\
& \sum_{j=0}^{N} h_{j i}^{d}+v_{i}^{d} \geq d_{i} \quad i=1,2, \ldots, N \\
& \sum_{j=0, j \neq i}^{N} \frac{h_{i j}^{d}+h_{i j}^{u}}{L C_{i j}}+\frac{h_{j i}^{d}+h_{j i}^{u} \leq 1}{L C_{j i}} \leq 0,1,2, \ldots, N \\
& f_{i j}^{u}+f_{i j}^{d} \leq B C_{i j} X_{i} X_{j} \\
& i=0,1,2, . ., N, j=0,1,2, . ., N, i \neq j \\
& \\
& \\
& \\
& \\
& \\
&
\end{array}
$$

The objective (1) minimizes the number of RSs and the total demand violations. The cost of an $\mathrm{RS}$ is $C$, while the penalty of demand violation is $P$ per bit per second. If we set $P \gg C$ and a feasible placement solution exists, the optimal solution will have negligible demand violations. Constraints (2) and (3) verify that the amount of traffic enters and exits the base station equals the total uplink and downlink traffic generated and received by the end users respectively. Constraints (4) and (5) verify the conservation of flow at each node. Constraints (6) and (7) verify that the uplink and downlink demands are met respectively. If the uplink or downlink demand is not satisfied at a particular node, a penalty will be imposed. Since local uplink and downlink traffic share the channel in a time-multiplexed fashion, constraint (8) verifies that the local traffic enters and exits through the $i^{\text {th }} \mathrm{RS}$ does not exceed its capacity. Finally, constraint (9) ensures that a positive backbone traffic exists between two nodes only if two RSs are installed at those nodes.

\subsection{Optimization by Bender's Decomposition}

Since the above mixed integer problem has a large number of continuous variables and a relatively small number of integer variables, it can be solved efficiently by Bender's decomposition. Bender's Method breaks down the problem to a sequence of small 0-1 integer problems which can be solved efficiently. In the following, we describe the algorithm that 
we used to solve the RS placement problem.

To apply Bender's decomposition to a mixed integer problem, all constraints have to be linear. Thus, we replace Constraint (9) by $f_{i j}^{u}+f_{i j}^{d} \leq B C_{i j} X_{i}$ and $f_{i j}^{u}+f_{i j}^{d} \leq B C_{i j} X_{j}$. The reformulated problem can now be cast in the following form ${ }^{1}$

$$
\begin{array}{ll}
\min _{\mathbf{X}, \mathbf{Y}} & c_{1} y+c_{2} x \\
\text { s.t. } & A_{1} y+A_{2} x \geq b,
\end{array}
$$

where $\mathbf{X}$ represents the set of integer variables $X_{i}$, and $\mathbf{Y}$ represents the set of continuous variables $f_{i j}^{u}, f_{i j}^{d}, h_{i j}^{u}, h_{i j}^{d}, v_{i}^{d}, v_{i}^{u}$. For a fixed value of the location variables $\widehat{x}$, problem (10) (11) reduces to the following demand violation problem:

$$
\begin{array}{ll}
\min _{\mathbf{Y}} & T(\mathbf{Y} \mid \widehat{x})=c_{1} y \\
\text { s.t. } & A_{1} y \geq b-A_{2} \widehat{x}
\end{array}
$$

Let us now consider the dual of the demand violation problem (12) (13). Let $\mathbf{U}$ be the set of dual variables. The dual of the demand violation problem may now be formulated as follows:

$$
\begin{array}{ll}
\max _{\mathbf{U}} & D(\mathbf{U} \mid \widehat{x})=\left(b-A_{2} \widehat{x}\right)^{t} u \\
\text { s.t. } & A_{1}^{t} u \leq c_{1}^{t}
\end{array}
$$

Denote the optimal solutions to the linear program (12) and (14) be $y^{*}$ and $u^{*}$ respectively. Then, by duality theory, $c_{1} y^{*}=\left(b-A_{2} \widehat{x}\right)^{t} u^{*}$.

We now consider all the extreme points of the dual problem (14) (15). Note that the extreme points are defined by the feasible region described by (15) which is independent of the location variables $\mathbf{X}$. Thus, the extreme points can be generated without any knowledge of the RS locations. Let us denote the $i^{t h}$ extreme point by $u^{i}$ and total number of extreme points be $p$. We know from the theory of linear programming that at least one optimal solution to any linear problem occurs at an extreme point of the feasible region. Thus, problem (10) (11) can be reformulate as the following pure $0-1$ problem:

$$
\begin{array}{ll}
\min _{\mathbf{X}} & D \\
\text { s.t. } & D \geq c_{2} x+\left(b-A_{2} x\right)^{t} u^{i} \quad \forall i \in[1, p] .
\end{array}
$$

The problem with the formulation $(16)(17)$ is that the number of extreme points of the dual problem is potentially very large. Thus, we do not want to enumerate all of the constraints in (17) explicitly. Also, at the optimal solution to (16)(17), only a small subset of the constraints (17) are likely to be tight. To generate the desired subset of extreme points, Bender's method adds constraints to the constraint set (17) iteratively [4]. Fig. 2 describes Bender's decomposition approach to solve the RS placement problem. In the next section, we discuss our numerical results.

\section{NUMERICAL ANALYSIS}

By using the proposed optimization framework, we evaluate the cost of deploying a heterogeneous wireless mesh network with relay stations in a sparse rural area where end users are distributed in a grid topology and the minimum distance between any two nodes is $1 \mathrm{~km}$. We set $C=1$

\footnotetext{
${ }^{1} a=b$ is equivalent to $a \geq b$ and $b \geq a$.
}

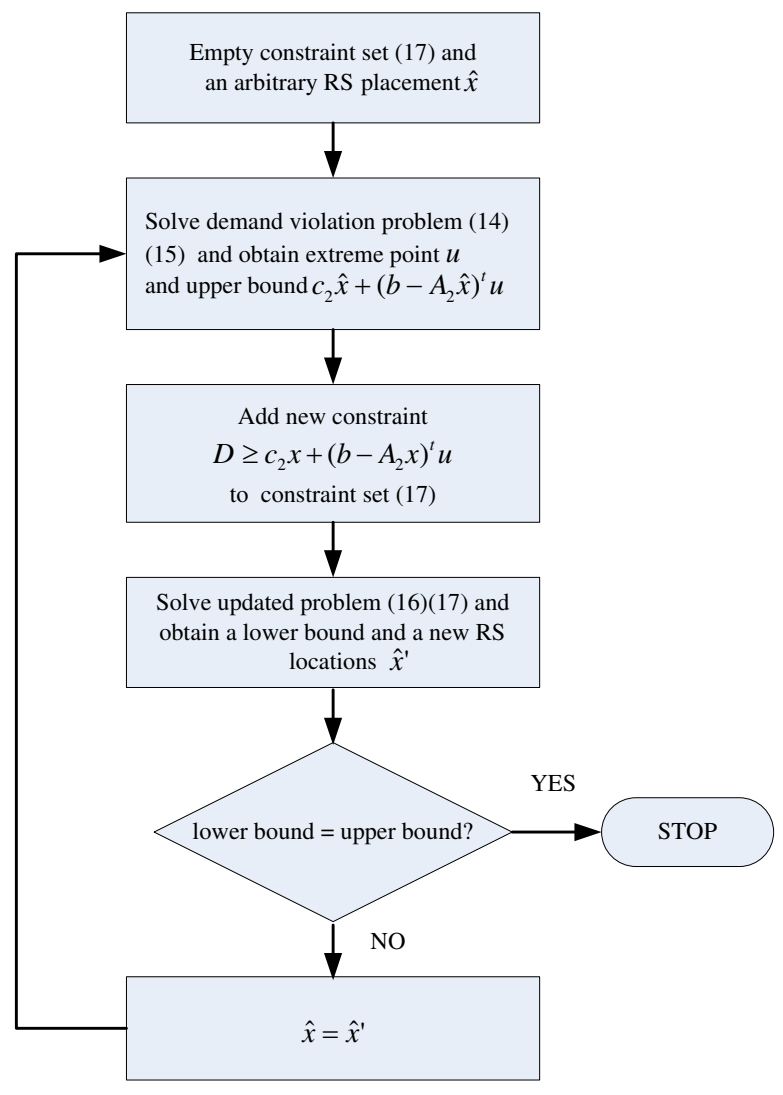

Figure 2: Flowchart of Bender's decomposition approach for solving the RS placement problem.

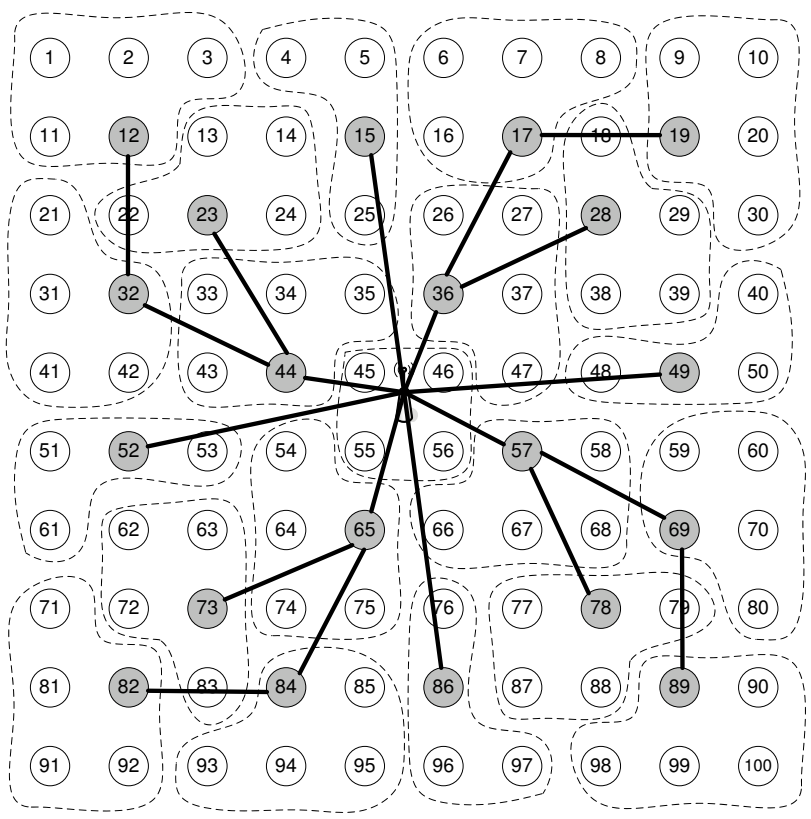

Figure 3: Network configuration of a heterogeneous mesh network with one local channel. 


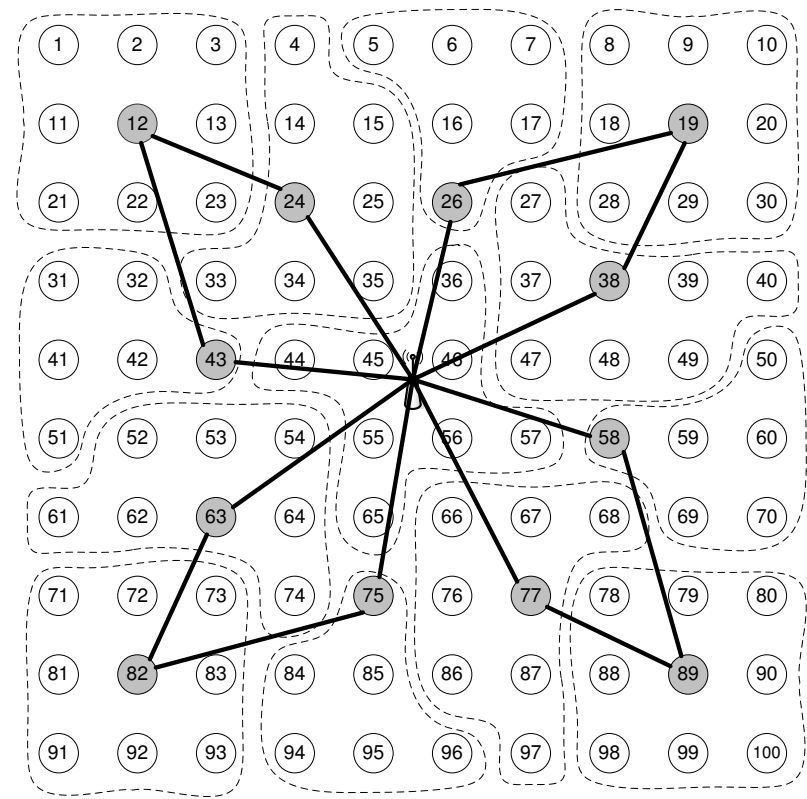

Figure 4: Network configuration of a heterogeneous mesh network with two local channels.

and $P=1000$. For the backbone networks, we use the IEEE 802.16 WirelessMAN-SC technology with transmitter reference power of $10 \mathrm{dBm}$, while for the local networks, we use the IEEE 802.16 WirelessMAN-OFDM technology with transmitter reference power of $15 \mathrm{dBm}$. The path loss exponent for the backbone and local networks are 2.8 and 2.4 respectively. Because of signal decay, bit rates are inversely proportional to distance. The rates are determined by the signal strength at the receiver and the thresholds specified by the IEEE 802.16 specifications. The backbone network occupies a $20 \mathrm{MHz}$ channel in the upper-microwave spectrum, while the local network occupies four $5 \mathrm{MHz}$ channels in the lower-microwave spectrum. The user downlink and uplink demands are $2 \mathrm{Mbps}$ and $1 \mathrm{Mbps}$ respectively. In the following, we discuss the effects of different number of local channel with respect to the minimum number of required RSs and their corresponding placements.

The optimal number of RSs required to satisfy the user demands are 20,12,8, and 8 for 1,2,3, and 4 local channels respectively. The network configurations for the networks with 1, 2 and 3 local channels are shown in Fig. 3, Fig. 4 , and Fig. 5 respectively. Intuitively, when the number of local channels available to each RS is high, an RS can satisfy the demands from more users. Hence the number of RSs required should be small. Fig. 3, Fig. 4, and Fig. 5 verify and further quantify this intuition. Besides this observation, when we further increase the number of local channel to 4 , the algorithm converge to the same value as the one with 3 channels. This suggests that for a given network topology and user demands, there exists a minimum number of required RSs regardless of the availability of other network resources.

\section{CONCLUSION}

In this work, we investigate the optimal placement of wireless relay stations to minimize the operational cost of a wireless mesh network. We presented a heterogeneous ar-

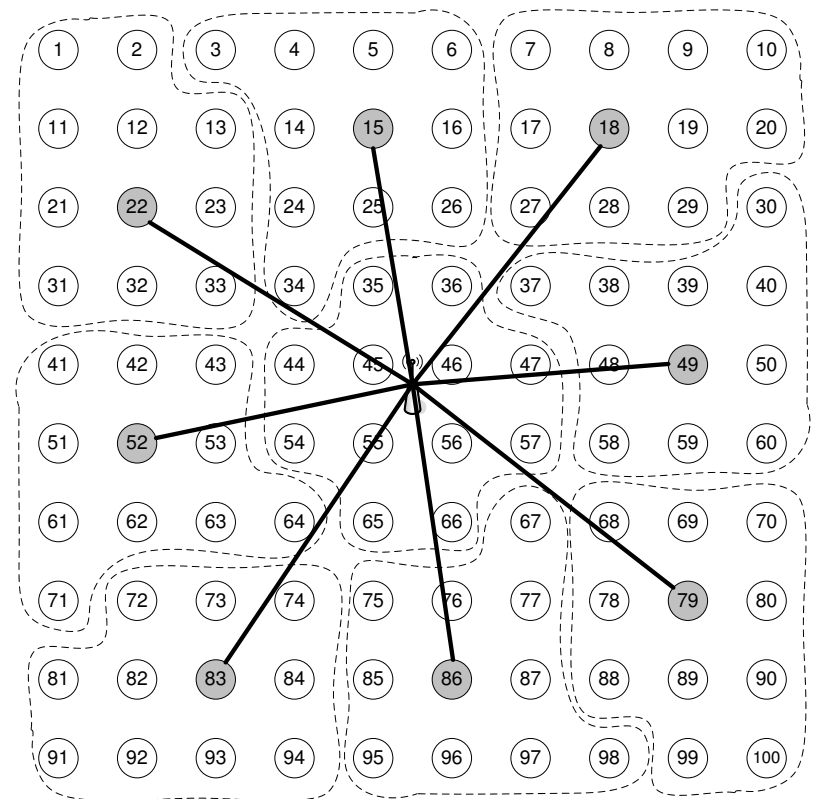

Figure 5: Network configuration of a heterogeneous mesh network with three local channels.

chitecture which uses relay stations to form a backbone and a local network. Furthermore, we have developed an analytical model to calculate the user demand violations with respect to different $\mathrm{RS}$ placement. We propose to use Bender's decomposition to compute the minimum number and placement of RSs of a heterogeneous wireless mesh network. Moreover, for different network environments, we quantify the effects of the number of local channels with respect to the optimal number and placement of RSs. Given a set of network parameters, the proposed framework and optimization technique can be used by network designers to compute the optimal placement of relay stations and provide design guidelines and network setup and maintenance cost estimations.

\section{REFERENCES}

[1] Y. Bejerano. Efficient integration of multihop wireless and wired networks with QoS constraints. IEEE Transactions on Networking, 12(6):1064- 1078, Dec. 2004.

[2] R. Chandra, L. Qiu, K. Jain, and M. Mahdian. Optimizing the placement of internet TAPs in wireless neighborhood networks. In Proc. of IEEE ICNP, pages 271 - 282, Berlin, Germany, Oct. 2004.

[3] Y. Chen, C. Chuah, and Q. Zhao. Sensor placement for maximizing lifetime per unit cost in wireless sensor networks. In Proc. of IEEE MILCOM, pages 1-6, Atlantic city, NJ, U.S.A., Oct. 2005.

[4] R. K. Martin. Large Scale Linear and Integer Programming. Kluwer Academic Publishers, 1 edition, 1999.

[5] A. So and B. Liang. A Lagrangian approach for the optimal placement of wireless relay nodes in wireless local area networks. In Proc. of the International IFIP-TC6 Networking Conference, pages 160-172, Coimbra, Portugal, May 2006. 